\section{Relationship between proximity to a cochlear implant center and early presentation in children with congenital hearing loss}

\author{
Yazeed A. Al-Shawi, MD, Fahad K. Alrawaf, MD, \\ Najd S. Al-Gazlan, MD, Munahi M. Al-Qabtani, MD, \\ Fida A. Almuhawas, MD, SB-ORL.
}

\section{ABSTRACT}

Objectives: To determine whether distance from a cochlear implant (CI) center can influences the ages of presentation and intervention for CI, which thus may influence CI outcomes in Saudi Arabia.

\begin{abstract}
Methods: This study comprised a retrospective crosssectional analysis of 221 patients who presented to the CI committee between March 2016 and March 2018 at King Abdullah Ear Specialist Center, Riyadh, Saudi Arabia. Through phone interviews and patients' files, age at suspicion, audiology testing, diagnosis, hearing aid fitting, and CI discussion were recorded for prelingually deaf children; additionally, demographic information were recorded. Patients were divided into 3 groups based on the distance between their place of residence and the nearest $\mathrm{CI}$ center (residing within $200 \mathrm{~km}, 200-500 \mathrm{~km}$, and $>500 \mathrm{~km}$ ).
\end{abstract}

Results: Patients living within $200 \mathrm{~km}$ showed the youngest mean age for all tested checkpoints; however, there were no statistically significant differences among the groups.

Conclusion: In Saudi Arabia, distance from CI center does not have a significant effect on age of presentation for CI intervention.

Keywords: cochlear implantation, congenital hearing loss, early presentation, proximity to CI center

Saudi Med J 2020; Vol. 41 (3): 314-317 doi: 10.15537/smj.2020.3.24918

Tormal hearing is an important sensory function 1 that enables children to develop communication skills. ${ }^{1}$ Congenital sensorineural hearing loss (SNHL) is a common disability in pediatric health, which can impede normal language development and educational achievement of deaf children. However, cochlear implant (CI) intervention has provided a remarkable solution. ${ }^{2}$ with results that vary widely among recipients. ${ }^{3}$ Early diagnosis of severe to profound SNHL is an important step in providing CI intervention at the recommended age to avoid language delay. ${ }^{1}$ Deaf children who received CI intervention before the age of 6 months performed better in expressive language and social skills later in school. ${ }^{4}$ Notably, post-CI results and language performance can be affected by many external factors that are related to the recipient's family. ${ }^{3}$ Although there is a higher number of $\mathrm{CI}$ candidates in rural populations than in urban populations, untreated SNHL is more prevalent in rural populations. ${ }^{5}$ Furthermore, rural populations have limited access to health care services, which can result in delayed diagnosis of SNHL, thereby negatively affecting outcomes of CI. Importantly, rural families must undertake extensive travel to tertiary medical centers to undergo hearing tests. ${ }^{1}$ Rehabilitation therapy after CI is a fundamental process for successful outcomes. ${ }^{2}$ However, rural families again experience difficulty because of the travel distance required to attain the full benefit of speech therapy sessions after CI. ${ }^{3}$ Thus, parents report dissatisfaction because of the shortage of speech and language therapy services provided in remote areas. ${ }^{6}$ Finally, the prevalence of CI mechanical complications or device malfunctions is reportedly higher among recipients in rural areas. ${ }^{2}$

The aim of this study is to determine whether among children with congenital SNHL, place of living and distance from a CI center influence the ages of presentation, diagnosis, hearing aid fitting, and discussion with CI committees. Secondary objectives include identification of the mean ages of suspicion of hearing loss, presentation to the hospital for audiological evaluation, hearing aid fitting, diagnosis, and discussion with CI committee, among the population in Saudi Arabia; moreover, this study aimed to study the delay before being fitted with hearing aids after establishing the diagnosis.

Methods. This cross-sectional retrospective study was performed in a tertiary hospital (King Abdullah Ear Specialist Center at King Abdulaziz University Hospital) in Riyadh, Saudi Arabia. The sample size in this study comprised 221 patients who presented to the CI committee at King Abdullah Ear Specialist Center for the first time during the period from March 2016 to March 2018 and fulfilled the following criteria. We included all pediatric patients with bilaterally severe to profound hearing loss who were prelingually deaf; we excluded any patient who was adult, who presented for the second ear, who had unilateral SNHL, who developed language pre-implant, or who had any psychological or neurological difficulties.

Using a custom-designed data collection sheet, we retrieved the following information through phone 
interviews with parents (or direct caregivers if parents were deceased) and patients' files: age at suspicion, age at audiology testing, age at diagnosis, age at hearing aid fitting, and age at CI discussion, as well as demographic information, place of residence, and distance from the nearest CI center. The data were stored as a hard copy, then entered in Excel sheet as a soft copy, then analyzed using IBM SPSS Statistics for Windows, version 22 (IBM Corp., Armonk, N.Y., USA). Patients were grouped as follows: Group 1 (those residing within 200 $\mathrm{km}$ of the CI center), Group 2 (those residing 200-500 $\mathrm{km}$ from the CI center), and Group 3 (those residing $>500 \mathrm{~km}$ from the CI center). We selected $200 \mathrm{~km}$ as a threshold because patients' families residing within 200 $\mathrm{km}$ could reasonably drive to the CI center in one day.

All demographic data were analyzed using frequency with percentages. Groups were compared using mean, and groups' different means were compared using one-way analysis of variance (ANOVA) and post-hoc test.

Formal review and approval were received by institutional review board of King Saud University. Confidentiality was maintained. The research was fully explained to each patient's family, and verbal informed consent was obtained from them during the phone interview for all patients included in this analysis.
Results. A total of 221 patients met the inclusion criteria for this study: 99 currently resided within $200 \mathrm{~km}$ of the CI center, 33 resided $200-500 \mathrm{~km}$ from the nearest CI center, and 89 resided $>500 \mathrm{~km}$ from the nearest CI center. Notably, 188 (85.1\%) of the patients' fathers were working and served as the main provider for the family, and $160(72.4 \%)$ of the patients' mothers were housewives without a job. Furthermore, 119 (54.8\%) of the patients' fathers and 109 (49.5\%) of the patients' mothers had a Bachelor's degree or higher for their level of education. A total of 64 (29\%) patients were the first child in their families, $51(23.1 \%)$ were the second, $33(14.9 \%)$ were the third, $28(12.7 \%)$ were the fourth, $16(7.2 \%)$ were the fifth, and $29(13.1 \%)$ were the sixth or higher. Additionally, 14 (6.5\%) of patients had no siblings, $48(22.1 \%)$ had only 1 sibling, 52 (24\%) had 2 siblings, 32 (14.7\%) had 3 siblings, 25 (11.5\%) had 4 siblings, 15 (6.9\%) had 5 siblings, and $31(14.3 \%)$ had $>6$ siblings.

The mean ages at suspicion of hearing loss, presentation to the hospital for audiological evaluation, hearing aid fitting, diagnosis, and discussion with CI committee are shown in Figure 1. The mean ages for the different groups, based on their distance from the CI center, are shown in Table 1.

A comparison of the mean ages at each stage among the 3 groups, using one-way analysis of variance (ANOVA), showed no significant differences ( $p$-values

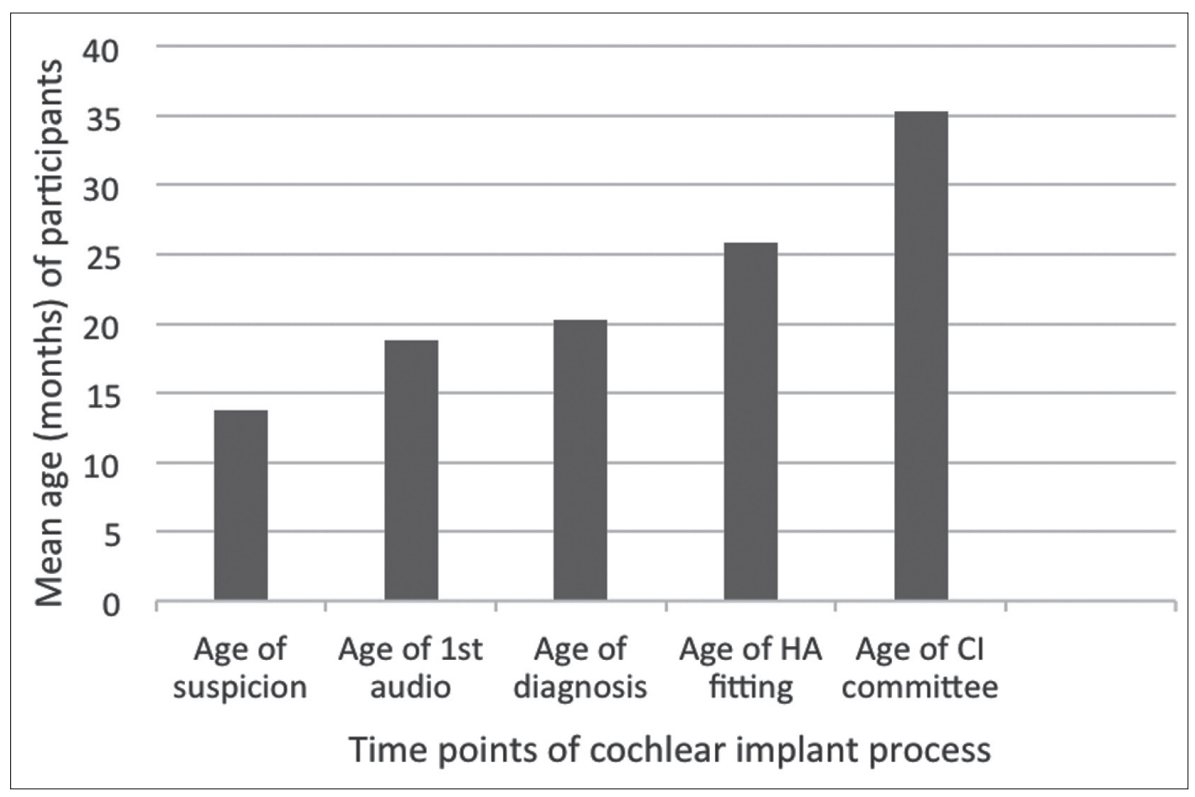

Figure 1 - Mean ages at presentation of patients for each stage of the cochlear implant process. HA - hearing aid 
Table 1 - Mean ages of presentation for different location groups (based on distance from cochlear implant center).

\begin{tabular}{|c|c|c|c|c|}
\hline Mean ages of presentation & $\mathrm{N}$ & Mean & Std. Deviation & Std. Error \\
\hline \multicolumn{5}{|l|}{ Age at suspicion by months } \\
\hline Residing within 200 km & 99 & 13.19 & 11.014 & 1.107 \\
\hline Residing $200-500 \mathrm{~km}$ away & 33 & 15.06 & 12.796 & 2.228 \\
\hline Residing $>500 \mathrm{~km}$ away & 89 & 13.93 & 16.848 & 1.786 \\
\hline Total & 221 & 13.77 & 13.849 & 0.932 \\
\hline \multicolumn{5}{|l|}{ Age at $1^{\text {st }}$ audio by months } \\
\hline Residing within 200 km & 99 & 16.82 & 12.718 & 1.278 \\
\hline Residing 200-500 km away & 33 & 22.61 & 19.190 & 3.341 \\
\hline Residing $>500 \mathrm{~km}$ away & 89 & 19.49 & 19.710 & 2.089 \\
\hline Total & 221 & 18.76 & 16.886 & 1.136 \\
\hline \multicolumn{5}{|l|}{ Age at diagnosis by months } \\
\hline Residing within $200 \mathrm{~km}$ & 99 & 18.29 & 13.692 & 1.376 \\
\hline Residing 200-500 km away & 33 & 24.18 & 20.229 & 3.521 \\
\hline Residing $>500 \mathrm{~km}$ away & 89 & 21.12 & 20.137 & 2.135 \\
\hline Total & 221 & 20.31 & 17.595 & 1.184 \\
\hline \multicolumn{5}{|l|}{ Age at HA fitting by months } \\
\hline Residing within 200 km & 99 & 23.24 & 14.481 & 1.455 \\
\hline Residing $200-500 \mathrm{~km}$ away & 33 & 29.52 & 21.902 & 3.813 \\
\hline Residing $>500 \mathrm{~km}$ away & 89 & 27.34 & 21.422 & 2.271 \\
\hline Total & 221 & 25.83 & 18.781 & 1.263 \\
\hline \multicolumn{5}{|c|}{ Age at cochlear implant committee } \\
\hline \multicolumn{5}{|l|}{ by months } \\
\hline Residing within 200 km & 97 & 32.10 & 25.042 & 2.543 \\
\hline Residing 200-500 km away & 31 & 40.16 & 26.431 & 4.747 \\
\hline Residing $>500 \mathrm{~km}$ away & 87 & 37.08 & 28.398 & 3.045 \\
\hline Total & 215 & 35.28 & 26.696 & 1.821 \\
\hline \multicolumn{5}{|c|}{ HA - hearing aid } \\
\hline
\end{tabular}

of age at suspicion, age at audiology testing, age at diagnosis, age at hearing aid fitting, and age at CI discussion are $0.792,0.204,0.214,0.156$, and 0.247 respectively). A subsequent post-hoc test also did not show any significant differences.

Discussion. Age at CI has been identified as an important predictor of language development outcomes in children with hearing loss. The location where the family's resides is a primary reason for delayed CI. ${ }^{7}$ There is a proportional relationship between age at CI and distance to the CI center, as access to care is more limited for patients who live in rural areas than for those who live in urban areas. ${ }^{8}$

As demonstrated in Table 1, the mean ages at each stage are comparable among the 3 groups $(p>0.05)$. This may be because of the availability of advanced hearing

Disclosure. Authors have no conflict of interests, and the work was not supported or funded by any drug company. assessment tools in peripheral hospitals, rapid access for patients living in the periphery to the closest CI center, and full governmental support to patients who are following up with tertiary hospitals outside their cities (namely, through airfare for patients and caregivers).

Regarding the mean ages at each stage of the CI process, we found that our patients do not meet the 2007 recommendations of the Joint Committee on Infant Hearing (JCIH). ${ }^{9}$ Notably, JCIH 2007 recommended that all infants are identified before the age of 3 months, and that early intervention (namely, hearing aids) should be initiated no later than 6 months of age. Therefore, initiatives are needed to increase general awareness of congenital hearing loss and the actions to take when it is suspected. Additionally, there is an unnecessary delay of approximately 5 months between confirmation of the diagnosis and fitting for hearing aids. The availability of hearing aids and facilities for fitting and servicing them is crucial to ensure early intervention for all children in both urban and rural areas.

Other factors that might have played a role in these findings are: the availability and abundance of 
flights from and to large cities in Saudi Arabia, the fact that familial awareness about hearing loss and its management is not affected by proximity to referral care centers (which might be secondary to the widespread of social networks), and the availability of appointments coordinators who help to make the visits of those coming from another city more convenient and productive by optimizing their schedule.

Study limitations. Mode of transportation was not taken into consideration; whether patients are coming to the CI center by car, train, or airplane. Although all patients with hearing loss have access to CI centers; other limitations are number of visits before being presented to the CI committee and how long did it take to be accepted after presentation, which were not taken into consideration as well.

In conclusion, in Saudi Arabia, the distance from residence to the nearest $\mathrm{CI}$ centers does not significantly impact the age of presentation to the hospital for hearing assessment. We encourage increased governmental logistic support for patients traveling to CI centers, as this may enable them to achieve comparable ages to their peers who reside near CI centers.

Acknowledgment. We would like to thank Editage for their efforts in English language editing.

Received 20th August 2019. Accepted 6th January 2020.

From the Department of Otolaryngology and Head and Neck Surgery, King Abdullah Ear Specialist Center, King Saud University, Riyadh, Kingdom of Saudi Arabia.
Address correspondence and reprints request to: Dr. Fida A. Almuhawas, Consultant, Department of Otolaryngology and Head and Neck Surgery, King Abdullah Ear Specialist Center, King Saud University, Riyadh, Kingdom of Saudi Arabia.E-mail: Fmuhawas@ksu.edu.sa

ORCID ID: https://orcid.org/0000-0002-3354-8756

\section{References}

1. Elpers J, Lester C, Shinn JB, Bush ML. Rural family perspectives and experiences with early infant hearing detection and intervention: A qualitative study. J Community Health 2016; 41: 226-233.

2. Noblitt B, Alfonso KP, Adkins M, Bush ML. Barriers to rehabilitation care in pediatric cochlear implant recipients. Otol Neurotol 2018; 39: e307-e313.

3. Sharma S, Bhatia K, Singh S, Lahiri AK, Aggarwal A. Impact of socioeconomic factors on paediatric cochlear implant outcomes. Int J Pediatr Otorhinolaryngol 2017; 102: 90-97.

4. Bush ML, Bianchi K, Lester C, Shinn JB, Gal TJ, Fardo DW, Schoenberg N. Delays in diagnosis of congenital hearing loss in rural children. J Pediatr 2014; 164: 393-397.

5. Chan S, Hixon B, Adkins M, Shinn JB, Bush ML. Rurality and determinants of hearing healthcare in adult hearing aid recipients. Laryngoscope 2017; 127: 2362-2367.

6. Zaidman-Zait A, Curle D, Jamieson JR, Chia R, Kozak FK. Cochlear implantation among deaf children with additional disabilities: Parental perceptions of benefits, challenges, and service provision. J Deaf Stud Deaf Educ 2015; 20: 41-50.

7. Fitzpatrick EM, Ham J, Whittingham J. Pediatric cochlear implantation: why do children receive implants late? Ear Hear 2015; 36: 688-694.

8. Bush ML, Burton M, Loan A, Shinn JB. Timing discrepancies of early intervention hearing services in urban and rural cochlear implant recipients. Otol Neurotol 2013; 34: 1630-1635.

9. Joint Committee on Infant Hearing. Year 2007 position statement: Principles and guidelines for early hearing detection and intervention programs. Pediatrics 2007; 120: 898-921. 